\title{
EFECTO DE LA SALINIDAD EN DOS PORTAINJERTOS DE VID CULTIVADOS A PIE FRANCO O INJERTADOS
}

\author{
EFFECT OF SALINITY ON TWO GRAFTED OR UNGRAFTED \\ GRAPE ROOTSTOCKS
}

\author{
Maritza Ojeda y Reinaldo Pire*
}

\author{
Postgrado de Horticultura, Decanato de Agronomía, Universidad Centroccidental "Lisandro Alvarado" (UCLA). Apartado 400. Barquisimeto 3001, Lara, \\ Venezuela. Fax: 58-251-2592571 \\ * Autor para correspondencia (rpire@ucla.edu.ve)
}

\section{RESUMEN}

La viticultura tropical se desarrolla en zonas de baja humedad donde el agua de riego frecuentemente presenta salinidad de mediana a alta. El objetivo de esta investigación fue evaluar la respuesta a la salinidad de los portainjertos de vid 'Criolla Negra' $y$ 'Villanueva' conducidos tanto a pie franco como injertados. Las plantas se cultivaron en recipientes de $5 \mathrm{~L}$ llenados con arena silícea y regadas con soluciones de $\mathrm{NaCl}+\mathrm{CaCl}_{2}$ (1:2), ajustadas a conductividades eléctricas (CE) de 0.75 (testigo), $2.25,3.75,5.25$ y $6.75 \mathrm{dS} \mathrm{m}^{-1}$. El material vegetal y los tratamientos salinos se evaluaron en arreglo factorial mediante un diseño completamente al azar con cinco repeticiones y dos plantas por unidad experimental. En condiciones de alta salinidad el crecimiento de la planta fue sólo $1 / 3$ de su crecimiento potencial, la densidad longitudinal de raíces disminuyó en $67 \%$ y la longitud específica en $38 \%$, la biomasa aérea en $64 \%$ y la radical en $58 \%$. La relación raíz/parte aérea de los materiales aumentó a medida que se incrementó el nivel salino. El portainjerto 'Criolla Negra', tanto a pie franco como injertado, resultó más afectado en valores altos de CE que 'Villanueva', y la supervivencia de portainjertos a pie franco fue superior en comparación con los materiales injertados. Los niveles de clorofila y el rendimiento cuántico fotosintético disminuyeron al aumentar la dosis salina. 'Villanueva' presentó el mayor contenido de prolina y promedió mayor biomasa y longitud radical. Los niveles de $\mathrm{K}$, Ca, Na y Cl tendieron a aumentar con la salinidad. El crecimiento de los injertos fue hasta $2 / 3$ menor al de los portainjertos a pie franco. La fisiología de los materiales se afectó a valores bajos de CE (0.75 a $2.25 \mathrm{dS}^{-1}$ ), pero el crecimiento vegetativo sólo se afectó con salinidades superiores (CE: 2.25 a $3.75 \mathrm{dS} \mathrm{m}^{-1}$ ).

Palabras clave: Vitis sp., crecimiento, portainjertos, salinidad.

\section{SUMMARY}

Tropical viticulture is developed in low humidity areas, where irrigation water frequently has medium to high salinity. The purpose of this study was to evaluate the response to salinity of the rootstocks 'Criolla Negra' and 'Villanueva', either grown on their own roots or grafted. The plants were grown in $5 \mathrm{~L}$ pots filled with silica sand, and irrigated with solutions of $\mathrm{NaCl}+\mathrm{CaCl}_{2}$ (1:2) adjusted to

Recibido: 26 de Agosto del 2010.

Aceptado: 2 de Febrero del 2011. electrical conductivities (EC) of 0.75 (control), $2.25,3.75,5.25$, and $6.75 \mathrm{dS} \mathrm{m}^{-1}$. The plant materials and saline levels were arranged in a factorial set of treatments in a complete randomized design, with five replications and two plants per plot. Salinity affected the vegetative growth starting at EC values from 2.25 to $3.75 \mathrm{dS} \mathrm{m}^{-1}$. Plant growth under high salinity was only $1 / 3$ of its potential, with associated decreases in root length density, of $38 \%$ in root specific length, of $64 \%$ in shoot biomass, and of $58 \%$ in root biomass. The root/shoot ratio increased as saline level was higher. 'Criolla Negra', either grafted or on its own roots, was more affected at higher EC than 'Villanueva', and survival of rootstocks on their own roots was higher than in grafted materials. Leaf chlorophyll and photosynthetic quantum yield decreased as the saline dose increased. 'Villanueva' showed the highest proline content and averaged highest biomass and root lenght. The levels of $\mathrm{K}, \mathrm{Ca}, \mathrm{Na}$ and $\mathrm{Cl}$ tended to increased with saline levels. Graft growth was only 2/3 of ungrafted rootstocks. The plant physiology was affected at low values of $\mathrm{EC}\left(0.75\right.$ to $\left.2.25 \mathrm{dS} \mathrm{m}^{-1}\right)$ but vegetative growth was affected only at higher values of salinity (EC: 2.25 to $3.75 \mathrm{dS} \mathrm{m}^{-1}$ ).

Index words: Vitis $s p$, growth, rootstocks, salinity.

\section{INTRODUCCIÓN}

En el trópico la vid (Vitis vinifera L.) produce entre dos y tres cosechas al año y se cultiva en zonas de baja humedad relativa para prevenir la incidencia de enfermedades fungosas; en zonas así de Venezuela es común la existencia de aguas de riego de mediana a alta salinidad (Villafañe et al., 1999). El exceso de sales en el suelo es uno de los principales problemas de producción de los cultivos en el mundo. La salinidad afecta el crecimiento de la planta por disminución en la absorción de agua, toxicidad de iones y desbalance nutricional (Willadino y Camara, 2004). El daño por salinidad depende principalmente de la concentración de las sales, del tiempo de exposición del cultivo y del genotipo (Hasegawa et al., 2000; Munns, 2002). Algunos efectos 
de la salinidad en plantas jóvenes de vid son: menor crecimiento vegetativo, menor captación de luz y acumulación de iones tóxicos (Fisarakis et al., 2001; Shani y Ben-Gal, 2005). El efecto del desbalance nutricional varía entre iones, y en vid los daños más importantes han sido reportados para $\mathrm{Cl}^{-}$y $\mathrm{Na}^{+}$(Fisarakis et al., 2001; Shani y Ben-Gal, 2005).

La vid se ha clasificada como un cultivo moderadamente sensible a salinidad, por lo que una de las estrategias de producción generalizada es el uso de portainjertos tolerantes (Pio et al., 2001), si bien existe una amplia variedad de tolerancia entre portainjertos y variedades de vid (Stevens et al., 1996; Cavagnaro et al., 2006). Un mecanismo de tolerancia para favorecer la absorción de agua en condiciones de alta salinidad consiste en el ajuste osmótico (Keller, 2005), especialmente con prolina (García y Medina, 2009).

Entre los cultivares de vid de importancia en la viticultura del Estado Lara en Venezuela se encuentran 'Criolla Negra' y 'Villanueva', dos genotipos criollos de vid que son o pueden ser usados como portainjertos. Olmo (1968) reportó a 'Criolla Negra' como un posible híbrido natural de Vitis vinifera $\mathrm{x} V$. caribaea, y se le considera un material rústico, moderadamente tolerante a déficit hídrico (Pire et al., 2007) y salinidad (Hidalgo, 1999), mientras que 'Villanueva' es conocido por su alto vigor y rápido crecimiento (Tortolero; Com. personal) ${ }^{1}$.

Debido a que el agua de riego en las zonas productoras de vid en el Estado Lara presentan problemas de salinidad y que las respuestas de los portainjertos señalados ante el estrés salino no han sido suficientemente estudiadas, el objetivo de este trabajo fue evaluar el efecto de la salinidad sobre el crecimiento vegetativo y aspectos fisiológicos de los portainjertos de vid 'Criolla Negra' y 'Villanueva', conducidos tanto a pie franco (sin injertar) como en condiciones de injertación.

\section{MATERIALES Y MÉTODOS}

El ensayo se condujo en un cobertizo de los Postgrados de Agronomía de la Universidad Centroccidental "Lisandro Alvarado" (UCLA), Cabudare, Estado Lara, Venezuela, ubicado a $10^{\circ} 01^{\prime} \mathrm{LN}$ y $500 \mathrm{msnm}$, con precipitación anual de $678 \mathrm{~mm}$, evaporación de $2174 \mathrm{~mm}$ y temperatura promedio máxima de $31.1{ }^{\circ} \mathrm{C}$ y mínima de $18.1^{\circ} \mathrm{C}$.

\footnotetext{
1 E Tortolero. Profesor de viticultura. Universidad Centroccidental "Lisandro Alvarado", Venezuela.
}

Las estacas de vid de 'Criolla Negra' y 'Villanueva' se colocaron a enraizar y fueron cultivadas durante 5 meses. Un grupo de ellas fue injertado con la variedad 'Red Globe' mediante el método de cuña lateral con doble lengüeta (Piña y Bautista, 2006). El injerto se dejó desarrollar durante 6 meses hasta obtener plantas de tamaño uniforme con un rango entre tres y cuatro brotes. A partir de este momento se inició la aplicación de los tratamientos salinos, los cuales se extendieron por 4 meses. Las plantas fueron cultivadas en bolsas de polietileno de $5 \mathrm{~L}$ y como sustrato se usó arena silícea.

Los tratamientos consistieron en cuatro materiales vegetales y cinco tratamientos salinos. Los materiales vegetales fueron los portainjertos 'Villanueva' y 'Criolla Negra', en dos formas: a pie franco e injertados, estos últimos en las combinaciones 'Red Globe'/'Villanueva' y 'Red Globe'/'Criolla Negra'. Los tratamientos de salinidad fueron soluciones ajustadas a las conductividades eléctricas (CE) de: T1: 0.75 (testigo); T2: 2.25; T3: 3.75; T4: 5.25; y $\mathrm{T} 5: 6.75 \mathrm{dS} \mathrm{m}^{-1}$ (máxima concentración salina de aproximadamente $40 \mathrm{mM})$, a partir de una solución madre de $\mathrm{NaCl}+\mathrm{CaCl}_{2}$ (1:2 de $\mathrm{Na}: \mathrm{Ca}$ ), según criterios de Shani y Ben-Gal (2005). El testigo representó la $\mathrm{CE}$ del agua de riego de la municipalidad y los tratamientos se aplicaron dos veces por semana durante cuatro meses.

Las plantas fueron fertilizadas semanalmente con 2.5 $\mathrm{g} \mathrm{L}^{-1}$ de fertilizante hidrosoluble $20-20-20\left(\mathrm{~N}: \mathrm{P}_{2} \mathrm{O}_{5}: \mathrm{K}_{2} \mathrm{O}\right)$ y micronutrimentos.

El ensayo se condujo como un factorial $4 \times 5$ (materiales de vid y niveles de salinidad) distribuidos en un diseño de completamente al azar, con cinco repeticiones, conformada cada una por dos unidades experimentales para un total de 10 plantas por tratamiento.

Transcurridos cuatro meses de la aplicación de los tratamientos salinos, las plantas se extrajeron de las bolsas de polietileno, se lavaron y se seccionaron en parte aérea y radical para la determinación de la materia seca, para luego colocarlas en una estufa de ventilación forzada a 75 ${ }^{\circ} \mathrm{C}$ hasta alcanzar peso constante. Con estas dos variables se determinó la relación raíz/parte aérea.

A partir de la materia seca de raíces se cuantificó la densidad longitudinal (Lv) (Pire et al., 1993); es decir, el cociente de la longitud de raíces finas (diámetro menor de $1 \mathrm{~mm}$ ) entre el volumen del sustrato $\left(5000 \mathrm{~cm}^{-3}\right)$. Luego de separarlas manualmente, estas raicillas fueron colocadas en la superficie plana de un escáner, y su imagen digitalizada mediante el programa SkyeRoot $\AA$, lo 
cual permitió la cuantificación de la longitud total mediante la sumatoria de todos los segmentos de raíz en la muestra. Adicionalmente, se determinó la longitud específica (LE) de las raíces al dividir la longitud entre su masa seca (Fitter, 1996).

Al final del ensayo se calculó la supervivencia como porcentaje de plantas vivas con respecto al número total de plantas por cada tratamiento.

Se determinó el contenido relativo de clorofila foliar con un medidor portátil de clorofila SPAD-502® marca Minolta, y de cada planta se obtuvo un promedio de tres hojas completamente expandidas del tercio medio del brote, seleccionadas al azar. Los valores se expresaron en unidades SPAD, y las mediciones se hicieron semanalmente.

La fluorescencia de clorofila foliar se determinó al final del ensayo con un fluorómetro modulado portátil marca Opti-Sciences modelo OS1-FL®, entre las 10 y las $13 \mathrm{~h}$. Se midió a través del parámetro rendimiento cuántico efectivo del fotosistema II (PSII), en hojas expuestas a la irradiancia. Esta variable se midió en dos hojas completamente expandidas del tercio medio del brote, seleccionadas al azar en una unidad experimental por tratamiento.

Se determinó el contenido de prolina en raíces al final del ensayo, para lo cual se pesó $0.1 \mathrm{~g}$ de tejido seco y se le agregó $10 \mathrm{~mL}$ de ácido sulfosalicílico a $3 \%(\mathrm{p} / \mathrm{v})$, y sobre el extracto obtenido se determinó la concentración del aminoácido con un espectrofotómetro Milton Roy Spectronic $21 \mathrm{D} \circledast$ a una longitud de onda de $520 \mathrm{~nm}$, utilizando tolueno como blanco según la metodología de Bates et al. (1973). La absorbancia se convirtió a concentración mediante una curva patrón de prolina elaborada con concentraciones conocidas.

Al final del ensayo se determinaron los contenidos de fósforo, potasio, calcio, magnesio, sodio y cloruro. Para estos análisis se colectaron muestras compuestas de 20 hojas en dos repeticiones por tratamiento. Las determinaciones de $\mathrm{P}, \mathrm{K}, \mathrm{Ca}$ y $\mathrm{Mg}$ se efectuaron en el laboratorio de Suelos del Decanato de Agronomía de la UCLA, mediante digestión seca y lectura por espectrofotometría de absorción atómica. El Na se analizó en el laboratorio de Suelos y Agua de Edafofinca (Turmero, Estado de Aragua) por digestión húmeda y lectura por fotometría de llama. El análisis de $\mathrm{Cl}^{-}$se efectuó en el laboratorio de Fisiología Vegetal del Postgrado de Agronomía de la UCLA, con un electrodo específico para $\mathrm{Cl}^{-}$(Orion 961700®), y se determinó en el peciolo de la hoja.
Los datos fueron procesados estadísticamente mediante análisis de varianza y separación de medias con la prueba de Tukey, con el programa Statistix versión $8 ®$. El contenido relativo de clorofila foliar se analizó mediante el procedimiento de mediciones repetidas.

\section{RESULTADOS Y DISCUSIÓN}

En general, la salinidad afectó en forma independiente las diferentes variables de crecimiento y fisiológicas de la vid, y no presentó efecto de interacción entre los diferentes materiales vegetales, con excepción del contenido de clorofila.

\section{Materia seca aérea y radical}

La materia seca aérea disminuyó conforme se incrementaron los niveles de salinidad, con diferencias $(\mathrm{P} \leq 0.05)$ a partir del rango de 2.25 a $3.75 \mathrm{dS} \mathrm{m}^{-1}$ en CE (Cuadro 1). La disminución de la materia seca aérea en los tratamientos más salinos (T4 y T5) en comparación con el testigo, fue en promedio de $64 \%$. La materia seca aérea del 'Villanueva' fue similar al del 'Criolla Negra', mientras que esta variable fue más afectada en el injerto 'Red Globe'/'Criolla Negra' que en 'Red Globe'/'Villanueva', con una reducción de casi $50 \%$.

La materia seca radical del tratamiento menos salino fue mayor $(\mathrm{P} \leq 0.05)$ que en los más salinos, con una diferencia de hasta $58 \%$ (Cuadro 1). El cociente raíz/parte aérea tendió a reducirse en los valores bajos de CE $\left(2.25 \mathrm{dS} \mathrm{m}^{-1}\right)$. En general, el comportamiento de la materia seca radical de los materiales fue similar a la de la parte aérea, ya que en ambos casos los portainjertos presentaron valores superiores en pie franco que en los materiales injertados; sin embargo, 'Villanueva' superó a 'Criolla Negra' en $26 \%$. La disminución de la materia seca radical de los portainjertos con respecto a sí mismos en condiciones de injertación, fue de $63 \%$ para 'Criolla Negra' y 56 \% para 'Villanueva'.

La acumulación de materia seca se usa ampliamente como una medida de crecimiento de la planta, porque refleja un balance entre la producción total de fotoasimilados y la respiración. Según Shani y Ben-Gal (2005), su reducción como consecuencia de la salinidad está asociada con disminuciones equivalentes en la absorción de agua debidas a un efecto osmótico, ya que la materia seca aérea declinó en $13 \%$ por cada incremento de $1.0 \mathrm{dS} \mathrm{m}^{-1}$ en la solución. 
Cuadro 1. Efecto de la salinidad en la materia seca aérea y radical, relación raíz/parte aérea, densidad longitudinal (Lv) y longitud específica (LE) de raíces, de dos portainjertos de vid cultivados a pie franco o injertados con 'Red Globe'.

\begin{tabular}{|c|c|c|c|c|c|c|}
\hline & Tratamientos & $\begin{array}{l}\text { Materia seca } \\
\text { aérea } \\
(\mathrm{g})\end{array}$ & $\begin{array}{l}\text { Materia seca } \\
\text { raíces } \\
(\mathrm{g})\end{array}$ & $\begin{array}{c}\text { Relación } \\
\text { raíz/parte aérea }\end{array}$ & $\begin{array}{c}\mathrm{LV} \\
\left(\mathrm{cm} \mathrm{cm}^{-3}\right)\end{array}$ & $\begin{array}{c}\mathrm{LE} \\
\left(\mathrm{cm} \mathrm{g}^{-1}\right)\end{array}$ \\
\hline \multirow{4}{*}{$\begin{array}{c}\text { Salinidad } \\
\left(\mathrm{CE}, \mathrm{dS} \mathrm{m}^{-1}\right)\end{array}$} & T1: 0.75 & $42.0 \mathrm{a}$ & $138.3 \mathrm{a}$ & $4.23 \mathrm{~b}$ & $3.75 \mathrm{a}$ & $355 \mathrm{a}$ \\
\hline & $\mathrm{T} 2: 2.25$ & $42.8 \mathrm{a}$ & $119.6 \mathrm{ab}$ & $3.30 \mathrm{~b}$ & $3.39 \mathrm{a}$ & $353 \mathrm{a}$ \\
\hline & T3: 3.75 & $31.7 \mathrm{~b}$ & $89.9 \mathrm{bc}$ & $4.22 \mathrm{~b}$ & $2.10 \mathrm{~b}$ & $316 \mathrm{ab}$ \\
\hline & T4: 5.25 & $15.2 \mathrm{c}$ & $48.3 \mathrm{~d}$ & $5.62 \mathrm{ab}$ & $1.13 \mathrm{c}$ & $231 \mathrm{ab}$ \\
\hline \multirow{5}{*}{ Material vegetal } & T5: 6.75 & $15.3 \mathrm{c}$ & $66.4 \mathrm{~cd}$ & $9.62 \mathrm{a}$ & $1.35 \mathrm{c}$ & $222 \mathrm{~b}$ \\
\hline & 'Villanueva' & $48.5 \mathrm{a}$ & $151.5 \mathrm{a}$ & $4.33 \mathrm{~b}$ & $3.86 \mathrm{a}$ & $282 \mathrm{a}$ \\
\hline & 'Criolla Negra' & $42.1 \mathrm{a}$ & $111.9 \mathrm{~b}$ & $3.17 \mathrm{~b}$ & $2.82 \mathrm{~b}$ & $256 \mathrm{a}$ \\
\hline & 'Red Globe'/'Villanueva' & $17.9 \mathrm{~b}$ & $65.2 \mathrm{c}$ & $4.93 \mathrm{~b}$ & $1.78 \mathrm{c}$ & $314 \mathrm{a}$ \\
\hline & 'Red Globe'/'Criolla Negra' & $9.1 \mathrm{c}$ & $41.4 \mathrm{c}$ & $9.16 \mathrm{a}$ & $0.92 \mathrm{~d}$ & $330 \mathrm{a}$ \\
\hline
\end{tabular}

Medias con letras iguales no son estadísticamente diferentes (Tukey, 0.05).

La menor acumulación de materia seca observada en los tratamientos salinos pudiera atribuirse al efecto osmótico que resulta de la elevada concentración de sales disueltas que disminuyen el potencial osmótico de la solución y consecuentemente la disponibilidad del agua para la planta (Willadino y Camara, 2004; Tadeo y Gómez-Cadenas, 2008). El déficit hídrico inducido por la salinidad restringe el crecimiento y ocasiona una menor tasa de división y elongación celular, así como disminución de la asimilación de $\mathrm{CO}_{2}$ (Pio et al., 2001).

Los resultados del ensayo aquí reportado son comparables con los señalados por Pio et al. (2001), quienes encontraron que la materia seca de cinco portainjertos de vid disminuyó proporcionalmente con los niveles de salinidad. Por su parte, Cavagnaro et al. (2006) reportaron respuestas diferenciales al someter a salinidad cultivares de vid europeos como 'Malbec', 'Cabernet Sauvignon' y 'Chardonnay' junto a cultivares criollos argentinos, ya que estos últimos presentaron mayores contenidos de materia seca.

En general, la relación raíz/parte aérea incrementó con la CE, de modo que el tratamiento más salino (T5) superó en más del doble a los menos salinos (Cuadro 1). La relación raíz/parte aérea refleja la distribución de la materia seca en la planta, y un valor mayor representa mayor vulnerabilidad de la parte aérea en relación a la raíz en condiciones de alta salinidad. Esta respuesta ha sido considerada como un mecanismo para disminuir la demanda respiratoria y el área de exposición ante estas condiciones (Medrano y Flexas, 2004; Tadeo y GómezCadenas, 2008), a la vez que puede reflejar una redistribución de asimilados hacia las raíces sometidas a este tipo de estrés (Willadino y Camara, 2004; Romero et al., 2004). Estos resultados son consistentes con los encontrados por Upreti y Murti (2010), quienes reportaron una mayor relación raíz/parte aérea en portainjertos de vid sometidos a tratamientos salinos, en relación con el testigo.

\section{Densidad longitudinal y longitud específica de raíces}

En general, la densidad longitudinal de raíces (Lv) disminuyó conforme se incrementaron los niveles de salinidad $(\mathrm{P} \leq 0.05)$, con una reducción promedio de 67 $\%$ en los tratamientos más salinos (T4 y T5), respuesta similar a la observada en la materia seca radical (Cuadro 1). La Lv fue mayor en 'Villanueva' que en 'Criolla Negra', y fue mayor en los materiales a pie franco que en los injertados.

La respuesta de la longitud específica de las raíces (LE) fue consistente con la Lv, ya que también disminuyó en la medida que incrementó la salinidad (Cuadro 1). Dado que la LE es indicativa de la longitud que posee un determinado segmento de raíz por unidad de masa, estos resultados indican que habría ocurrido un aumento del grosor de las raicillas como respuesta a la salinidad, tal como fue señalado por Fitter (1996).

Existen evidencias que el estrés salino afecta negativamente al crecimiento de las raíces; sin embargo, puede haber un incremento de ellas a niveles moderados de salinidad, lo que puede estar relacionado con un mecanismo de aclimatación por ajuste osmótico (Romero et al., 2004).

Ojeda y Pire (1996) encontraron que en condiciones de campo la mayor Lv de 'Criolla Negra' ocurre en los primeros $45 \mathrm{~cm}$ del perfil del suelo, lo cual indica que la mayor cantidad de raíces activas de la planta está propensa a sufrir daños por salinidad. Por otra parte, los valores de Lv encontrados en este ensayo son superiores a los reportados por Pire y Díez (2006), lo cual se atribuye a que estos autores trabajaron en suelos con alta proporción de poros pequeños, a diferencia del presente ensayo donde se utilizó sustrato de arena con alta proporción de poros grandes que facilitan el crecimiento y proliferación de raíces (Richards, 1983). 


\section{Supervivencia de plantas}

La supervivencia de las plantas disminuyó con el incremento de los niveles de salinidad (Cuadro 2). El portainjerto 'Villanueva', tanto a pie franco como injertado, empezó a verse afectado a menores valores de CE que el portainjerto 'Criolla Negra', a la vez que la supervivencia de los portainjertos a pie franco fue superior que en los injertados.

Se ha reportado que la supervivencia de las plantas de vid puede variar en función del portainjerto, cultivar, nivel de salinidad y tiempo de exposición, tanto en condiciones controladas in vitro (Cavagnaro et al., 2006) como en campo (Shani y Ben-Gal, 2005). En tal sentido, Cavagnaro et al. (2006) reportaron que materiales criollos argentinos sobrevivieron más a la salinidad que los cultivares europeos. Shani y Ben-Gal (2005) señalaron que la mortalidad de plantas de vid 'Sugraone' aumentó linealmente con la salinidad una vez que comenzó a verse afectada por los altos niveles de $\mathrm{Cl}^{-}$.

Es importante señalar que en este ensayo, previo a la muerte de las plantas se observó necrosis en los bordes de las hojas, seguida por un quemado y defoliación, lo cual se atribuyó al posible efecto tóxico de las sales (Bernstein et al., 1969).

\section{Contenido relativo de clorofila foliar}

La salinidad afectó significativamente el contenido de clorofila de los portainjertos 'Villanueva' y 'Criolla Negra' $(P \leq 0.05)$. Los contenidos al inicio del ensayo fueron comparables entre todos los materiales, con un promedio de 31.7 unidades SPAD (Figura 1); en el portainjerto 'Villanueva' hubo un comportamiento similar entre los tratamientos de salinidad hasta aproximadamente 60 d después de iniciar la aplicación de los mismos, y después los valores empezaron a disminuir en T4 y T5. Al final del ensayo, el contenido de clorofila disminuyó en la medida que aumentó la CE del agua de riego, con el mayor valor para T1 y el menor para T5, con grupos estadísticos intermedios para el resto de los tratamientos de salinidad. La disminución promedio en T5 fue de $46 \%$ con respecto al $\mathrm{T} 1$.

En el cv. 'Criolla Negra' los valores fueron similares entre los diferentes niveles de salinidad durante los primeros $15 \mathrm{~d}$; a partir de ese momento, en los tratamientos más salinos los contenidos de clorofila empezaron a descender, tendencia que se mantuvo hasta el final del ensayo. A los 120 d se observó que el contenido de clorofila foliar fue menor en la medida que incrementaron los niveles de salinidad, con el mayor valor para $\mathrm{T} 1$ y el menor para $\mathrm{T} 5$, aunque este último fue significativamente igual al resto de los tratamientos de salinidad; la disminución promedio fue de $42 \%$ con respecto al T1. En la Figura 1 se muestra que en ambos cultivos el contenido de clorofila varía a través del tiempo y que para 'Criolla Negra' existe una menor separación entre las líneas, lo que permite inferir que este portainjerto fue menos afectado por los tratamientos de salinidad, en comparación con 'Villanueva'.

La clorofila foliar de los materiales injertados fue poco afectada por la salinidad en comparación con los materiales a pie franco, y al final del ensayo no se detectaron diferencias entre los tratamientos salinos (Figura 1).

La salinidad genera senescencia foliar que va acompañada de una removilización de nutrientes, y una consecuente disminución del contenido de clorofila antes de la abscisión de la hoja (Keller, 2005; Quesada y Valpuesta, 2008). El hecho de que los materiales injertados mantuvieron un rango de clorofila foliar similar tanto en el tiempo como entre los tratamientos salinos, pudiera estar asociado con su menor vigor y crecimiento (Cuadro 1). En tal sentido, una menor tasa de crecimiento implicaría menor demanda y menos movilización de nutrientes, lo cual podría retardar la aparición de los síntomas cloróticos (Gárate y Bonilla, 2008).

Cuadro 2. Efecto de la salinidad sobre el porcentaje de supervivencia de dos portainjertos de vid cultivados a pie franco o injertados con 'Red Globe' $(n=10)$.

\begin{tabular}{|c|c|c|c|c|c|}
\hline \multirow{2}{*}{ Material vegetal } & \multicolumn{5}{|c|}{ Tratamiento de salinidad (CE, $\left.\mathrm{dS} \mathrm{m}^{-1}\right)$} \\
\hline & T1: 0.75 & T2: 2.25 & T3: 3.75 & T4: 5.25 & T5: 6.75 \\
\hline 'Villanueva' & 100 & 100 & 90 & 90 & 77 \\
\hline 'Criolla Negra' & 100 & 100 & 100 & 63 & 70 \\
\hline 'Red Globe'/'Villanueva' & 100 & 86 & 86 & 86 & 63 \\
\hline 'Red Globe'/'Criolla Negra' & 100 & 100 & 83 & 60 & 40 \\
\hline
\end{tabular}



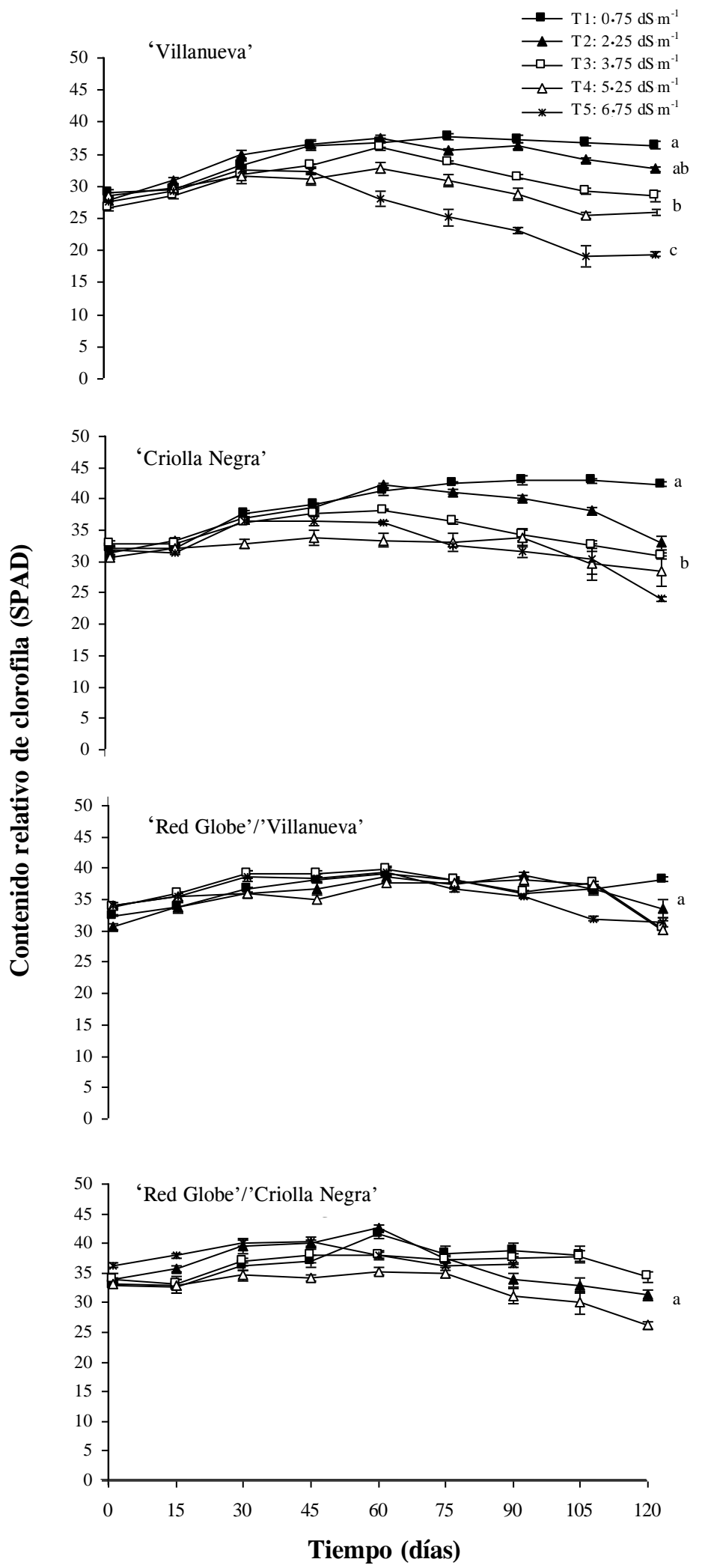

Figura 1. Efecto de la salinidad sobre el contenido relativo de clorofila foliar de dos portainjertos de vid cultivados a pie franco o injertados con 'Red Globe' 


\section{Fluorescencia de clorofila foliar}

El rendimiento cuántico fotosintético del fotosistema II (PSII) fue afectado por los tratamientos de salinidad $(\mathrm{P} \leq 0.05)$, donde $\mathrm{T} 1$ dio el mayor valor y $\mathrm{T} 4 \mathrm{y} \mathrm{T} 5$ dieron los menores, con una reducción cercana a $18 \%$ (Cuadro 3), lo cual sugiere una posible fotoinhibición foliar. No se detectaron diferencias significativas entre los materiales vegetales, aunque se observó una ligera tendencia en 'Villanueva' a superar a 'Criolla Negra', tanto a pie franco como injertada.

La fluorescencia de clorofila se ha usado como una valiosa herramienta no destructiva para determinar la tolerancia al estrés por salinidad, porque produce un estimado de la fotoinhibición foliar en términos de la tasa de transporte lineal del electrón durante la fotosíntesis (Baker et al., 2007). En plantas moderadamente tolerantes a la salinidad se ha reportado hasta $25 \%$ de reducción del rendimiento de la fluorescencia de clorofila (Azcón-Bieto et al., 2008). Este rendimiento cuántico se correlaciona con la asimilación de $\mathrm{CO}_{2}$ (Hikosaka et al., 2004), y bajo condiciones de estrés salino el desbalance entre la captación de luz para la fotosíntesis y la utilización de reductores para la fijación de $\mathrm{CO}_{2}$ puede traducirse en un daño al fotosistema II o fotoinhibición (Hasegawa et al., 2000).

\section{Contenido de prolina en raíces}

En esta variable no se detectaron diferencias significativas entre los tratamientos, aunque el contenido de prolina en las raíces tendió a incrementarse ligeramente en la medida que aumentó la CE del agua de riego (Cuadro 3). Entre los materiales vegetales sí hubo diferencias $(\mathrm{P} \leq 0.05)$; los mayores valores se detectaron en 'Villanueva' tanto a pie franco como injertado, en comparación con 'Criolla Negra', lo que refleja que el primer portainjerto acumuló mayores contenidos del aminoácido en condiciones naturales.

Aunque la prolina no pudo ser determinada en las hojas debido a la defoliación que ocurrió en los tratamientos más salinos, se ha demostrado que los niveles de este aminoácido en las raíces, aunque un poco menores, son proporcionales a las concentraciones foliares (García y Medina, 2009). Las raíces pueden realizar ajuste osmótico mediante la acumulación de azúcares y aminoácidos, entre los que se destaca la prolina (Willadino y Camara, 2004; Romero et al., 2004), lo cual disminuye el potencial osmótico de la raíz y de esta manera favorece la absorción del agua que es limitada por la salinidad. Sin embargo, Cramer et al. (2007) reportaron que en plantas de vid es más común el aumento de la prolina por efecto del estrés hídrico que por efecto de la salinidad.

La mayor acumulación natural del aminoácido en 'Villanueva' podría explicar la tendencia general de este material a superar en biomasa y longitud radical a 'Criolla Negra', ya que la prolina puede servir como sustrato respiratorio o actuar en el balance de nitrógeno (Hasegawa et al., 2000).

\section{Nutrición mineral}

En general, 'Villanueva' tendió a presentar valores de fósforo un poco más altos que 'Criolla Negra', lo cual podría atribuirse a una condición genética de los portainjertos ya que no se observó una tendencia definida con relación a los tratamientos salinos (Cuadro 4). En otros estudios, la respuesta del fósforo en la vid sometida a condiciones salinas ha sido variable; por ejemplo, Stevens et al. (1996) señalaron que la salinidad incrementa el contenido foliar de $\mathrm{P}$, mientras que Bernstein et al. (1969) no pudieron detectar un efecto consistente en este sentido.

Con relación al contenido foliar de potasio se observó que este elemento tendió a incrementar en ambos portainjertos a medida que aumentó la salinidad (Cuadro 4). Stevens et al. (1996) y Grattan y Grieve (1999) sugirieron que entre los mecanismos involucrados en este tipo de respuesta hay una elevada tasa de transporte desde las raíces, o por intercambio de $\mathrm{K}^{+}$por $\mathrm{Na}^{+}$. 
Cuadro 3. Efecto de la salinidad sobre la fluorescencia de clorofila foliar en términos del rendimiento cuántico fotosintético del fotosistema II (PSII), y contenido radical de prolina en dos portainjertos de vid cultivados a pie franco o injertados con 'Red Globe'.

\begin{tabular}{|c|c|c|c|}
\hline & Tratamientos & $\begin{array}{l}\text { Rendimiento cuántico } \\
\text { fotosintético del PSII }\end{array}$ & $\begin{array}{l}\text { Contenido de prolina en raíces } \\
\text { ( } \mu \text { mol de prolina/g peso seco) }\end{array}$ \\
\hline \multirow{3}{*}{$\begin{array}{l}\text { Salinidad } \\
\left(\mathrm{CE}, \mathrm{dS} \mathrm{m}^{-1}\right)\end{array}$} & T1: 0.75 & $0.77 \mathrm{a}$ & $3.35 \mathrm{a}$ \\
\hline & $\mathrm{T} 2: 2.25$ & $0.69 \mathrm{ab}$ & $3.80 \mathrm{a}$ \\
\hline & T4: 5.25 & $0.66 \mathrm{~b}$ & $3.91 \mathrm{a}$ \\
\hline \multirow{3}{*}{$\begin{array}{c}\text { Material } \\
\text { vegetal }\end{array}$} & T5: 6.75 & $0.63 \mathrm{~b}$ & $4.03 \mathrm{a}$ \\
\hline & 'Villanueva' & $0.71 \mathrm{a}$ & $4.52 \mathrm{a}$ \\
\hline & 'Red Globe'/'Criolla Negra' & $0.68 \mathrm{a}$ & $2.99 \mathrm{c}$ \\
\hline
\end{tabular}

Medias con letras iguales no son estadísticamente diferentes (Tukey, 0.05).

Cuadro 4. Efecto de la salinidad sobre la concentración mineral foliar de dos portainjertos de vid cultivados a pie franco $(n=2)$.

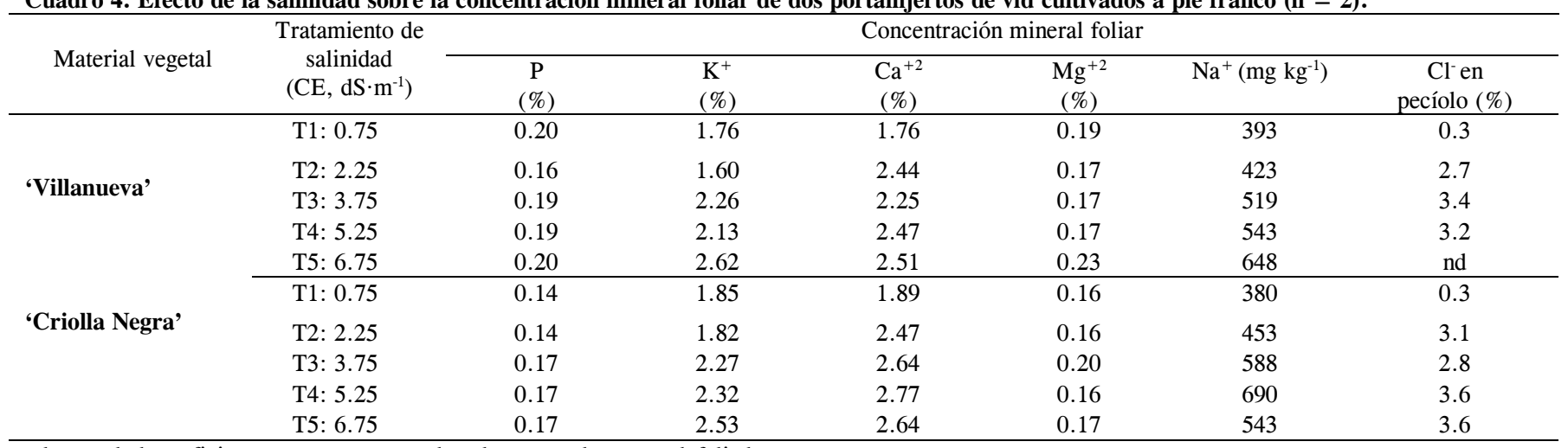

nd $=$ no hubo suficiente muestra por que las plantas estaban muy defoliadas.

Una respuesta similar ocurrió con el contenido de calcio, el cual tendió a aumentar en la medida que se incrementaron los tratamientos de salinidad (Cuadro 4), atribuible al efecto de la adición de este elemento en los tratamientos salinos. Según Grattan y Grieve (1999), la presencia de $\mathrm{Ca}^{+2}$ en el sustrato influye en la selectividad de $\mathrm{K}^{+} / \mathrm{Na}^{+}$, lo cual favorece la absorción de $\mathrm{K}^{+}$a expensas del $\mathrm{Na}^{+}$, especialmente en genotipos tolerantes a la salinidad; esto podría explicar la tendencia aquí observada antes señalada del incremento del $\mathrm{K}^{+}$foliar. Por otra parte, el $\mathrm{Ca}^{+2}$ ayuda a mantener la integridad de la membrana, y por tanto disminuye la pérdida de $\mathrm{K}^{+}$en las células de la raíz.

Los contenidos foliares de magnesio no mostraron tendencia alguna en relación con los tratamientos de salinidad ni tampoco entre portainjertos (Cuadro 4). Este resultado es contrario a los reportados en otros trabajos, en los que se encontró que la concentración foliar de $\mathrm{Mg}^{+2}$ disminuye por efecto de la salinidad (Bernstein et al., 1969; Stevens et al., 1996). Es posible que en nuestro ensayo la frecuente aplicación de solución nutritiva al sustrato haya evitado la disminución del contenido foliar de magnesio y de otros elementos minerales.
En general, los contenidos de $\mathrm{Na}^{+}$incrementaron proporcionalmente con los tratamientos de salinidad, sin diferencias apreciables entre ambos portainjertos (Cuadro 4). Downton (1985) señaló que un efecto principal de la salinidad sobre la vid es un desbalance entre $\mathrm{Na}^{+}$y $\mathrm{K}^{+}$, generado por su antagonismo, lo cual no pudo observarse aquí. En otros casos, la alta concentración de $\mathrm{Na}^{+}$puede también interferir en la actividad de muchas enzimas (Bartels y Sunkar, 2005).

Los contenidos de cloruros tendieron a incrementar con los tratamientos de salinidad, con promedios similares en ambos portainjertos (Cuadro 4). Shani y Ben-Gal (2005) reportaron que la concentración de $\mathrm{Cl}^{-}$y $\mathrm{Na}^{+}$en las hojas incrementó con los tratamientos de salinidad, y que este incremento fue moderado en CE de 0.5 a $7 \mathrm{dS} \mathrm{m}^{-1}$, rango similar al utilizado en el ensayo que aquí se describe.

Los genotipos tolerantes a la salinidad pueden usar varios mecanismos para prevenir la acumulación de $\mathrm{Na}^{+}$y $\mathrm{Cl}^{-}$, entre los cuales están restringir su absorción mediante transportadores específicos de la membrana (Hasegawa et $a l .$, 2000), el secuestro de iones en las vacuolas, la regulación de su transporte hacia la parte aérea (Fisarakis et al., 2001; Storey et al., 2003), o su exclusión hacia el 
exterior (Hasegawa et al., 2000). Asimismo, podrían distribuir la acumulación de $\mathrm{Cl}^{-}$hacia otros órganos de la planta (Stevens et al., 1996). La muerte de varias plantas aquí observada ante los más altos niveles salinos permiten suponer que hubo una inadecuada regulación de los mecanismos de tolerancia de los genotipos en condiciones de elevada concentración de $\mathrm{Cl}^{-}$y $\mathrm{Na}^{+}$.

En resumen, el conjunto de variables evaluadas sugieren que el principal efecto de las salinidad sobre la planta fue el osmótico, mediante el cual las sales disueltas disminuyen el potencial osmótico de la solución del suelo y consecuentemente la disponibilidad del agua para la planta. En menor grado pudo haber ocurrido un efecto iónico que se habría manifestado al final del ensayo en los tratamientos más salinos, representado por una acumulación excesiva de los iones $\mathrm{Cl}^{-}$y $\mathrm{Na}^{+}$.

\section{CONCLUSIONES}

El crecimiento de los portainjertos injertados fue menor al de los cultivados a pie franco. En general, la fisiología de los materiales de vid se afectó desde valores de conductividad eléctrica del agua de riego en el rango entre 0.75 y $2.25 \mathrm{dS} \mathrm{m}^{-1}$, y se acentuó a valores superiores de salinidad $\left(2.25\right.$ y $\left.3.75 \mathrm{dS} \mathrm{m}^{-1}\right)$. El crecimiento de la planta en condiciones de alta salinidad fue de sólo un tercio de su crecimiento potencial. Independientemente del nivel salino, el portainjerto 'Villanueva' superó en biomasa y longitud radical al 'Criolla Negra'.

\section{AGRADECIMIENTOS}

Al Consejo de Desarrollo Científico Humanístico y Tecnológico de la Universidad Centroccidental "Lisandro Alvarado" (CDCHT-UCLA), por el financiamiento mediante el proyecto 008-AG-2005, así como al Instituto de la Uva de la UCLA por el suministro del material vegetal. Igualmente, a la Lic. María Castillo y al Br. José Valdez por la participación en la recolección de datos.

\section{BIBLIOGRAFÍA}

Azcón-Bieto J, I Fleck, X Aranda, N Gómez-Casanovas (2008) Fotosíntesis: factores ambientales y cambio climático. In: Fundamentos de Fisiología Vegetal. J Azcón-Bieto, M Talón (eds). McGraw-Hill Interamericana. Madrid. pp:247-263.

Baker N, J Harbinson, D Kramer (2007) Determining the limitations and regulation of photosynthetic energy transduction in leaves. Plant Environ. 30:1107-1125.

Bartels D, R Sunkar (2005) Drought and salt tolerance in plants. Crit. Rev. Plant Sci. 24:23-58.

Bates L, R Waldren, D Teare (1973) Rapid determination of free proline for water-stress studies. Plant Soil 39:205-207.
Bernstein L, C F Ehlig, R A Clark (1969) Effect of grape rootstocks on chloride accumulation in leaves. J. Amer. Soc. Hort. Sci. 94:584-590.

Cavagnaro J B, M T Ponce, J Guzmán, M A Cirrincione (2006) Argentinean cultivars of Vitis vinifera grow better than european ones when cultures in vitro under salinity. Biocell 30:1-7.

Cramer G R, A Ergül, J Grimplet, R L Tillett, E A R Tattersall, M C Bohlman, D Vincent, J Sonderegger, J Evans, C Osborne, D Quilici, K A Schlauch, D A Schooley, J C Cushman (2007) Water and salinity stress in grapevines: early and late changes in transcript and metabolites profiles. Funct. Integr. Genomics 7:111-134.

Downton W J S (1985) Growth and mineral composition of the Sultana grapevine as influenced by salinity and rootstock. Aust. J. Agric. Res. 36:425-434.

Fisarakis I, J Chartzoulakis, D Stavrakas (2001) Response of Sultana vines (Vitis vinifera L.) on six rootstocks to $\mathrm{NaCl}$ salinity exposure and recovery. Agric. Water Manage. 51:13-27.

Fitter A (1996) Characteristics and functions of root systems. In: Plant Roots, The Hidden Half. Y Waisel, A Eshel, U Kafkafi (eds). Marcel Dekker. N.Y. pp:1-20.

Gárate A, I Bonilla (2008) Nutrición mineral y producción vegetal. In: Fundamentos de Fisiología Vegetal. J Azcón-Bieto, M Talón (eds). McGraw-Hill Interamericana. Madrid. pp:143-164.

García M, E Medina (2009) Acumulación de iones y solutos orgánicos en dos genotipos de caña de azúcar, estresados con sales simples o suplementadas con calcio. Bioagro 21:3-14.

Grattan S R, C M Grieve (1999) Salinity-mineral nutrient relations in horticultural crops. Sci. Hort. 78:127-157.

Hasegawa P, R Bressan, J-K Zhu, H Bohnert (2000) Plant cellular and molecular responses to high salinity. Annu. Rev. Plant Physiol. Plant Mol. Biol. 51:463-499.

Hidalgo L (1999) Tratado de Viticultura General. Ed. Mundi-Prensa. Madrid. $1172 \mathrm{p}$.

Hikosaka K, M Kato, T Hirose (2004) Photosynthetic rates and partitioning of absorbed light energy in photoinhibited leaves. Physiol. Plant. 121:699-708.

Keller M (2005) Deficit irrigation and vine mineral nutrition. Amer. J. Enol. Vitic. 56:267-283.

Medrano H, J Flexas (2004) Respuesta de las plantas al estrés hídrico. In: La Ecofisiología Vegetal, Una Ciencia de Síntesis. M Reigosa, N Pedrol, A Sánchez (eds). Thomson Editores Spain. Madrid. pp:253-286.

Munns R (2002) Comparative physiology of salt and water stress. Plant Cell Environ. 25:239-250.

Ojeda M, R Pire (1996) Densidad de raíces en vid sometidas a diferentes láminas de riego por goteo. Proc. Interam. Soc. Trop. Hort. 40:219-223.

Olmo H P (1968) The potential for a grape and wine industry in Venezuela. Mimeografiado CBR. Caracas. $30 \mathrm{p}$.

Piña S, D Bautista (2006) Evaluación del crecimiento vegetativo de cultivares de vid para mesa bajo condiciones de trópico semiárido de Venezuela. Rev. Fac. Agron. (LUZ) 23:405-416.

Pio V A, C Horst Bruckner, H E Prieto Martínez, C A Martínez y Huaman, P R Mosquim (2001) Características fisiológicas de porta-enxertos de videira em solucão salina. Sci. Agric. 58:139143.

Pire R, J Díez (2006) El crecimiento de las raíces de vid en la condición de trópico $\left(10^{\circ} 01^{\prime} \mathrm{N}\right)$ de Tarabana, estado Lara, Venezuela. Rev. Fac. Agron. (LUZ) 23:453-463.

Pire R, A Yépez, A Urdaneta (1993) Densidad de raíces de un árbol de mango en la Estación Experimental de la UCLA en Tarabana. Bioagro 5:63-69.

Pire R, A Pereira, J Díez, E Fereres (2007) Evaluación de la tolerancia a la sequía de un portainjerto venezolano de vid y posibles mecanismos condicionantes. Agrociencia 41:435-446. 
Quesada M A, V Valpuesta (2008) Senescencia y abscisión. In: Fundamentos de Fisiología Vegetal. J Azcón-Bieto, M Talón (eds). McGraw-Hill Interamericana. Madrid. pp:559-576.

Richards D (1983) The grape root system. Hort. Rev. 5:127-168.

Romero C, A Espinosa-Ruíz, M C Cutanda, C Cortina, P Hernández, F A Culiáñez-Maciá (2004) La osmoregulación: mecanismos y significado. In: La Ecofisiología Vegetal, Una Ciencia de Síntesis. M Reigosa, N Pedrol, A Sánchez (eds). Thomson Editores. Spain. Madrid. pp:603-620.

Shani U, A Ben-Gal (2005) Long-term response of grapevines to salinity: osmotic effects and ion toxicity. Amer. J. Enol. Vitic. 56:148-154

Stevens R, G Harvey, G Davies (1996) Separating the effects of foliar and root salt uptake on growth and mineral composition of four grapevine cultivars on their own roots and on Ramsey rootstock. J. Amer. Soc. Hort. Sci. 121:569-575.

Storey R, D P Schachtman, M R Thomas (2003) Root structure and cellular chloride, sodium and potassium distribution in salinized grapevines. Plant Cell Environ. 26:789-800.
Tadeo F R, A Gómez-Cadenas (2008) Fisiología de las plantas y el estrés. In: Fundamentos de Fisiología Vegetal. J Azcón-Bieto, M Talón (eds). McGraw-Hill Interamericana. Madrid. pp:577597.

Upreti KK, GSR Murti (2010) Response of grape rootstocks to salinity: changes in root growth, polyamines and abscisisc acid. Biol. Plant. 54:730-734.

Villafañe R, O Abarca, M Azpúrua, T Ruiz, J Dugarte (1999) Distribución espacial de la salinidad en los suelos de Quíbor y su relación con las limitaciones de drenaje y la calidad del agua. Bioagro 11:43-50.

Willadino L, T Camara (2004) Origen y naturaleza de los ambientes salinos. In: La Ecofisiología Vegetal, Una Ciencia de Síntesis. M Reigosa, N Pedrol, A Sánchez (eds). Thomson Editores Spain. Madrid. pp:303-300. 